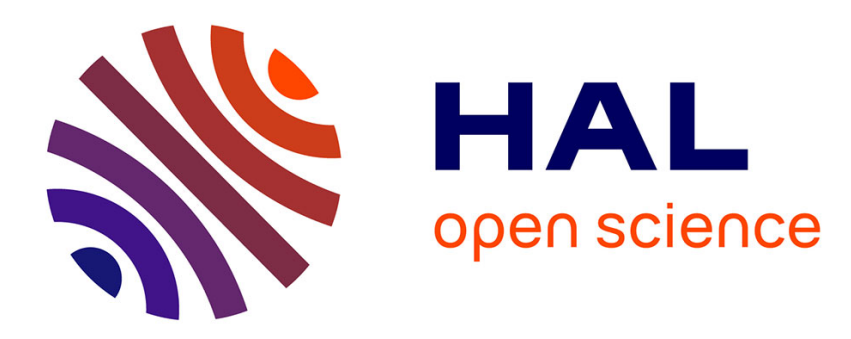

\title{
Le Parlement et la Cour des comptes
}

Pascal Jan

\section{To cite this version:}

Pascal Jan. Le Parlement et la Cour des comptes. Pouvoirs - Revue française d'études constitutionnelles et politiques, 2013, Le Renouveau du Parlement, 146, pp.107-116. 10.3917/pouv.146.0107. halshs-00999436

\section{HAL Id: halshs-00999436 \\ https://shs.hal.science/halshs-00999436}

Submitted on 4 Jan 2022

HAL is a multi-disciplinary open access archive for the deposit and dissemination of scientific research documents, whether they are published or not. The documents may come from teaching and research institutions in France or abroad, or from public or private research centers.
L'archive ouverte pluridisciplinaire HAL, est destinée au dépôt et à la diffusion de documents scientifiques de niveau recherche, publiés ou non, émanant des établissements d'enseignement et de recherche français ou étrangers, des laboratoires publics ou privés. 


\section{PARLEMENT \\ ET COUR DES COMPTES}

ierre Joxe, Philippe Seguin, Didier Migaud, des personnalités politiques ayant fait une longue carrière et exercé de hautes responsabilités parlementaires, ont été propulsés Premier président de la Cour des comptes au cours des vingt dernières années. Les relations de la haute juridiction financière avec le Parlement ne peuvent qu'en être affectées positivement. Mais les liens les unissant ne se limitent pas à cet aspect. Ils ne font que prolonger assez logiquement une relation ancienne qui n'a cessé de se renforcer en s'accélérant ces deux dernières décennies dans un climat de revalorisation de l'institution parlementaire.

Le Parlement français entretient depuis le XIX siècle, au travers de régimes constitutionnels différents donc, des rapports singuliers avec la Cour des comptes. Tout commence à la Restauration. La loi du 14 septembre 1822 remet à la Cour des comptes la responsabilité de certifier l'exactitude des comptes généraux publiés par le ministre des Finances après que la loi du 27 juin 1819 oblige la Cour à assister les chambres. Cette compétence n'a jamais été exploitée par les parlementaires pour accroître leur emprise sur l'exécutif, tout comme n'a pas donné lieu à développements la transmission aux chambres d'un rapport annuel (loi du 21 avril 1832 qui annonce le futur rapport public). Â la décharge du Parlement, ces aspects du contrôle budgétaire ne constituaient pas le cour des préoccupations des libéraux et des partisans du régime parlementaire, encore trop peu nombreux. Cependant, les relations existent entre le Parlement et la Cour des comptes, et c'est assez naturellement que la Constitution du 27 octobre 1946 consacre la mission d'assistance de la Cour au Parlement et que la Constitution de 1958 reprend à son compte cette avancée constitutionnelle en son article 47, dernier alinéa. Mais force est d'admettre que la prescription constitutionnelle 
ne se traduit pas par l'approfondissement du contrôle parlementaire. Là encore, le contexte de méfiance, voire de défiance à l'égard des élus de la nation, n'est guère propice à l'exploitation de cette faculté offerte aux députés et sénateurs.

Un changement majeur intervient dans le courant des années 1990. De plus en plus de voix s'élèvent pour réclamer une revalorisation du Parlement, un renforcement de ses prérogatives de contrôle. Si la jurisprudence du Conseil constitutionnel avait depuis quelques années déjà desserré l'étau constitutionnel pesant sur le domaine législatif, beaucoup restait à faire pour la fonction de contrôle. Les modifications des règlements de l'Assemblée nationale et du Sénat interviennent pour traduire cette aspiration, néanmoins limitées dans leur portée par les contraintes constitutionnelles auxquelles veille scrupuleusement 108 et obligatoirement le Conseil constitutionnel. C'est donc du constituant et du législateur tant organique qu'ordinaire que vont surgir les bouleversements majeurs qui aujourd'hui caractérisent les relations entre le Parlement et la Cour des comptes. Le mode de collaboration entre ces institutions s'inscrit toujours dans le cadre de l'assistanat des juges financiers aux parlementaires. Au-delà de ce constat qui n'apporte rien d'autre à la réflexion que la simple lecture de la Constitution, l'assistance de la Cour au Parlement s'inscrit en réalité dans une alliance recherchée par le Parlement et à son profit, mais dans le respect de l'indépendance de l'institution de la rue Cambon.

\section{LA COUR DES COMPTES,}

ALliéE Du PARLEMENT CONTRÔLEUR DE L'EXÉCutif

C'est au début du second septennat de François Mitterrand (1988-1995) que les parlementaires, toutes opinions politiques confondues, multiplient les revendications d'un renforcement du pouvoir parlementaire dans son activité de contrôle de l'exécutif. La négociation du futur traité de Maastricht constitue de ce point de vue un moment révélateur. Ce qui n'est pas discutable, c'est le souci des députés et des sénateurs de consolider leurs prérogatives en matière financière en s'attachant presque systématiquement l'appui de la Cour des comptes, qui apparaît comme une alliée privilégiée. L’assistance de la Cour est recherchée comme gage de sérieux d'un contrôle plus poussé des finances publiques nationales, et ce n'est pas un paradoxe si la matière budgétaire et financière constitue le cœur de la reconquête du pouvoir législatif, matière qui a toujours constitué la colonne vertébrale du contrôle parlementaire, de son émancipation à 
son affirmation. Contrôler les finances publiques, c'est aussi contrôler les politiques gouvernementales.

Le premier acte - même s'il est toujours arbitraire de fixer un point de départ - de cette reconquête du pouvoir parlementaire est la loi du 7 avril 1991 qui valide une initiative de la Cour s'autorisant à publier des rapports "thématiques» par une interprétation osée d'une loi de 1967 qui prévoit la possibilité d'accompagner le rapport annuel de fascicules « distincts», sans autre précision. Par cette loi, les parlementaires manifestent leur intention de contrôler plus efficacement l'exécution des politiques publiques et recherchent alors l'expertise de la Cour des comptes.

Sur le plan constitutionnel, la première étape fut la loi du 22 février 1996 instituant les lois de financement de la sécurité sociale. Cette révision de la Constitution répond à une revendication parlementaire qui est apparue dans la décennie précédente et qui concerne un aspect essentiel des finances publiques. L'ajout d'un article (47-1) dans la Constitution qui étend la collaboration de la Cour des comptes et du Parlement aux finances sociales prolonge en réalité une initiative parlementaire qui, par la loi du 27 juillet 1994, oblige la Cour à établir un rapport sur l'état de la sécurité sociale et à faire part des résultats de son contrôle aux assemblées. L'article 13 de cette dernière loi disposait notamment que, «chaque année, la Cour des comptes transmet au Parlement un rapport analysant les comptes de l'ensemble des organismes de sécurité sociale soumis à son contrôle et faisant une synthèse des avis émis par les comités départementaux d'examen des comptes de la sécurité sociale, éventuellement complété par ses observations aux autorités de tutelle et les réponses à celles-ci ». L'article LO 132-3 du code des juridictions financières $(\mathrm{CJF})$ adopté à la suite de la révision constitutionnelle oblige donc la Cour à présenter en septembre un rapport sur l'application des lois de financement de la sécurité sociale, soit en amont de la discussion parlementaire du projet de cette loi qui débute en octobre.

Le domaine des finances sociales a été investi par le Parlement qui a recherché ouvertement le soutien de la Cour pour en examiner et contrôler les principaux aspects (objectifs de recettes et de dépenses). Mais l'étape décisive d'une nouvelle relation entre la Cour et les assemblées intéresse les finances générales de l'État. Les parlementaires soucieux de moderniser les outils de la gestion publique et de renforcer leur contrôle vont agir sur les trois leviers législatifs possibles, constitutionnel, organique et ordinaire, sans oublier d'en préciser les termes dans le règlement de leurs assemblées respectives. 
Le pas décisif, pour ne pas dire le big-bang budgétaire, intervient avec l'adoption consensuelle de la loi organique relative aux lois de finances (LOLF). D'initiative parlementaire, la LOLF du $1^{\text {er }}$ août 2001 constitue le socle de ce renouveau des relations entre la Cour et le Parlement. La Constitution, à l'occasion de la révision de son cinquantenaire, prolonge les acquis législatifs donnant une nouvelle dimension à la mission constitutionnelle d'assistance de la Cour en y intégrant l'évaluation des politiques publiques. Désormais, la Constitution lui fait une place à part entière en son article 47-2 intégré dans son titre $\mathrm{V}$ consacré aux rapports entre le Parlement et le gouvernement: « La Cour des comptes assiste le Parlement dans le contrôle de l'action du gouvernement. Elle assiste le Parlement et le gouvernement dans le contrôle de l'exécution des lois de finances et de l'application des lois de financement de la sécurité sociale ainsi que dans l'évaluation de politiques publiques [...]».

De la combinaison de ces textes il résulte que le Parlement oblige la Cour des comptes à lui transmettre systématiquement et automatiquement un certain nombre de documents et données, et se réserve aussi la possibilité de solliciter la Cour ponctuellement sans possibilité pour elle de s'y soustraire. À cette fin, la loi organique du $1^{\text {er }}$ août 2001, notamment en son article 58, redéfinit et élargit la mission d'assistance de la Cour des comptes au Parlement.

Outre le rapport annuel de la Cour qui désormais est l'objet d'un débat parlementaire, la loi organique prévoit la transmission de nombreux rapports destinés à alimenter la réflexion des parlementaires et à les seconder dans l'exercice du contrôle de l'activité gouvernementale. Sans qu'il soit nécessaire d'opérer une hiérarchie entre les divers documents, on peut citer en premier lieu les rapports directement en lien avec les lois de finances. Ainsi, la Cour livre ses analyses sur l'exécution des crédits par mission et par programme indispensables dans l'examen des lois de règlement. Elle évalue la pertinence des enveloppes globalisées et fongibles mises à la disposition des ministres. La Cour produit également un rapport préliminaire au rapport sur l'évolution de l'économie nationale et sur les orientations des finances publiques qui structure le débat d'orientation budgétaire. En prolongement de ces travaux, elle analyse les modifications budgétaires opérées par les décrets d'avance, renforçant ainsi considérablement le principe de l'autorisation budgétaire. Plus exactement, tout projet de loi de finances est accompagné d'un rapport de la Cour des comptes sur les mouvements de crédits opérés par voie administrative dont la ratification est demandée dans le projet de loi de finances rectificative ou de règlement. Par ailleurs, la Cour exerce 
depuis 2007 la certification de la régularité, de la sincérité et de la fidélité des comptes de l'État et de ceux du régime général de la sécurité sociale. À cette fin, elle établit un rapport à l'attention du Parlement, document de référence indispensable pour examiner l'exécution des crédits de l'État. Le Parlement peut de cette manière vérifier la conformité des états financiers de l'État à un ensemble de règles et de principes budgétaires et financiers. Il en va de la crédibilité et de la fiabilité des comptes de l'État français dans un monde globalisé où les économies nationales sont sujettes à des évaluations d'organismes étrangers et indépendants, et dont les observations orientent, par exemple, la capacité à emprunter des États. Compte tenu de l'importance de ces certifications pour le respect de la sincérité et de la régularité des comptes dont doivent s'assurer politiquement les parlementaires, il n'est pas surprenant que le constituant ait tenu en 2008 à inscrire dans le marbre constitutionnel cette nouvelle fonction. Tous ces rapports forment l'ossature de l'assistance de la Cour à la fonction de contrôle parlementaire qui, elle-même, a bien entendu une incidence sur la capacité d'initiative législative des élus.

En dehors de ces rapports, les parlementaires ont souhaité contraindre la Cour à leur transmettre d'autres documents censés alimenter leur information et, par voie de conséquence, leur fonction de contrôle de l'action gouvernementale. À ce titre, et sans qu'il soit nécessaire de les répertorier tous, on peut mentionner certains rapports thématiques comme les «bleus » intéressant les comptes et la gestion des entreprises publiques (art. L. 135-3 CJF) ou encore la transmission des référés (L. 135-5 CJF). Jusqu'à l'intervention de la loi de finances rectificative pour 1995, les commissions des finances étaient exceptionnellement destinataires de ces référés, car dépendantes de la bonne volonté du Premier ministre. Dès 1996, la transmission est obligatoire au terme d'un délai de six mois si les observations des magistrats restaient sans réponse ministérielle et, depuis l'intervention de la LOLF, la transmission des référés et des réponses est automatique dans un délai ramené à trois mois. Cette information adressée aux commissions des finances des assemblées parlementaires suscite des questions écrites, voire justifie certaines auditions et propositions de loi. Pour s'en tenir à un exemple récent, ce fut le cas en 2011 pour le référé sur la politique universitaire qui, après l'audition de la ministre, déboucha sur l'adoption d'une initiative législative parlementaire.

Toute cette activité d'assistance de la Cour est imposée par le législateur. La LOLF a également entendu élargir les hypothèses d'intervention de la Cour en consacrant des dispositifs où les parlementaires 
sont demandeurs d'informations ponctuelles et non plus systématiques. L'une des dispositions phares de la loi organique, qui a connu de très nombreuses applications tant à l'Assemblée nationale qu'au Sénat, est la faculté offerte aux commissions des finances et à celles en charge des affaires sociales de réclamer des enquêtes à la Cour des comptes. C'est le signe d'une détermination parlementaire à exercer totalement et pleinement le contrôle parlementaire budgétaire. Ici également, l'efficacité du contrôle s'appuie sur l'expertise de la Cour des comptes. L'article 58-2 de la LOLF prévoit ainsi «la réalisation de toute enquête demandée par les commissions de l'Assemblée nationale et du Sénat chargées des finances sur la gestion des services ou organismes qu'elle contrôle. Les conclusions de ces enquêtes sont obligatoirement communiquées dans un délai de huit mois après la formulation de la demande à la commission dont elle émane, qui statue sur leur publication ». Franc succès de cette procédure comparée à celle figurant à l'article 58-1 qui oblige la Cour à répondre aux demandes d'assistance formulées par le président et le rapporteur général de la commission chargée des finances, qui usent peu de cette faculté d'assistance d'un parlementaire dans le cadre de ses missions de contrôle et d'évaluation. Les demandes d'enquête formulées à la Cour n'ont cessé de se multiplier. Le flux ne devrait pas se tarir. De 2001 à 2013, près de cent dix demandes ont été adressées à la Cour, témoignant de l'intérêt porté à la procédure mais plus encore de son utilité pour les parlementaires. Le succès de cette procédure, qui existait déjà dans l'arsenal législatif, s'explique assurément par le «plus» de la loi organique qui enserre le travail dans un délai de huit mois et par la publicité qui est faite de ces travaux par les commissions des finances. C'est un ticket gagnant-gagnant, d'autant plus que depuis 2010 les enquêtes de la Cour sont accompagnées de recommandations dont s'emparent les parlementaires. Le succès appelant le succès, pareille évolution est constatée pour le contrôle des finances sociales. L'article LO 132-3-1 CJF prévoit un dispositif semblable mais plus restrictif dans son champ d'application et surtout non assorti d'un délai de remise de conclusions d'enquête. Ces circonstances expliquent certainement une moindre sollicitation de la Cour (trois demandes par an contre dix pour l'article 58-2), mais n'enlèvent rien à l'intérêt que suscite l'exploitation de ses travaux, qui se poursuivent également par des auditions des rapporteurs de la Cour des comptes et des représentants des administrations ou organismes concernés.

Enfin, il convient de mentionner le Comité d'évaluation et de contrôle (CEC), nouvel organe parlementaire ad hoc de l'Assemblée nationale qui 
prend la suite de plusieurs organismes comme l'Office parlementaire d'évaluation des politiques publiques, institué par une loi du 14 juin 1996 mais supprimé quatre ans plus tard. L'intérêt du CEC est de procéder à des évaluations dont le champ dépasse le périmètre d'une seule commission permanente (art. 146-3 du règlement de l'Assemblée nationale) comme l'évaluation, en 2012, des politiques de lutte contre le tabagisme ou celle des politiques en faveur de la création d'entreprises, sans compter la place faite aux groupes parlementaires de l'opposition et minoritaires qui disposent notamment d'un droit de «tirage » annuel. Dans ce cadre, le CEC peut solliciter l'assistance de la Cour dont les conclusions alimentent ses réflexions sur l'évolution nécessaire d'une législation ou la nécessité de poser des questions aux ministres concernés. Mais, au-delà, c'est une véritable collaboration qui s'est instaurée, puisque les rapporteurs de la Cour et les rapporteurs parlementaires s'invitent mutuellement dans leurs organismes respectifs pour rendre compte de leurs travaux.

\section{LA COUR, UNE ALLIÉE IN DÉPENDANTE, GAGE D'UN CONTRÔLE PARLEMENTAIRE DE QUALITÉ}

L'assistance de la Cour des comptes au Parlement est au cour de la revalorisation du contrôle parlementaire et plus globalement de la fonction des députés et sénateurs, qui y trouvent matière à réflexion pour leurs initiatives législatives. Cette assistance est d'autant plus fructueuse que la Cour jouit d'une indépendance reconnue, qui donne d'autant plus de force à la livraison de ses analyses et synthèses à destination des parlementaires. C’est un point essentiel de la relation Parlement-Cour des comptes. Sans cette garantie de rang constitutionnel, il eût été fort probable que toutes les avancées constitutionnelles, législatives et conventionnelles fussent demeurées très en deçà des espérances initiales. Et il faut rendre gré au Conseil constitutionnel d'avoir veillé à cette indépendance dans le cadre de son contrôle obligatoire des lois organiques ${ }^{1}$ et des règlements des assemblées parlementaires. L'ancien rapporteur général et présidente de chambre Claire Basy-Malaurie a fort bien mis en perspective cet aspect. Concluant un colloque sur les rapports de la Cour des comptes au Parlement en 2007, la désormais conseillère constitutionnelle souligne le souci d'indépendance des magistrats financiers, gage d'une «information pertinente» livrée aux parlementaires: «Pour peser avec efficacité sur la décision, le Parlement devait bénéficier d'une information pertinente,

1. CC, 2001-448 DC, 25 juillet 2001. 
c'est-à-dire portant sur des résultats significatifs, mais aussi précision, information que je qualifierai par une expression, peut-être très familière, de vérité des prix. Tâche dans laquelle la Cour excelle puisque notamment au travers de ses rapports sur l'exécution de la loi de finances, mais aussi de tous ses rapports, elle peut dire, de manière fiable et impartiale, les chiffres qu'on vous donne sont les bons ${ }^{2}$...»

Dans sa version initiale, la LOLF en son article 58 comportait un alinéa obligeant la Cour à transmettre aux présidents et aux rapporteurs généraux des commissions parlementaires en charge des finances publiques son programme de travail, avec faculté pour les élus de formuler des avis et de communiquer leurs demandes d'enquête. Le Conseil constitutionnel saisi obligatoirement du texte organique invalida cette disposition en se fondant sur l'indépendance reconnue aux juridictions administratives.

114 Le juge donne plein effet à la théorie de l'équidistance. La Cour étant une juridiction administrative dont l'indépendance est établie par les principes fondamentaux reconnus par les lois de la République, le législateur organique ne pouvait contraindre la Cour à soumettre le contenu de ses activités, même non juridictionnelles, à supervision parlementaire. Les relations informelles établies entre la Cour et le Parlement n'autorisent pas ce dernier à s'immiscer dans les travaux de la première. Surtout, le juge constitutionnel précise qu'il «appartiendra à la Cour des comptes de faire en sorte que l'équilibre voulu par le constituant ne soit pas faussé au détriment de l'un de ces deux pouvoirs », c'est-à-dire le Parlement ou le gouvernement, conseillé également par la Cour en vertu de la Constitution.

Le juge constitutionnel eut l'occasion de réitérer sa doctrine lors de la modification du règlement de l'Assemblée nationale précisant les règles de fonctionnement du CEC. Dans sa décision du 25 juin $2009^{3}$, le Conseil constitutionnel indique «qu'il n'appartient pas au règlement mais à la loi de déterminer les modalités selon lesquelles un organe du Parlement peut demander» l'assistance de la Cour. La loi du 3 février 2011 a précisé en conséquence les modalités présidant à cette relation entre la Cour et le CEC. Sur cette base, certains sujets sont exclus d'une demande d'assistance à la Cour. Tel est le cas d'une demande de suivi ou de contrôle de l'exécution des lois de finances en raison de leur encadrement par d'autres textes. Au-delà de cette observation, il importe

2. Claire Basy-Malaurie, «Les rapports de la Cour des comptes au Parlement: synthèse », Revue française de finances publiques, $\mathrm{n}^{\circ}$ 99, 2007.

3. CC, 2009-581 DC, 25 juin 2009. 
d'insister sur un point mis en relief avec l'illustration précédente: l'utilité des travaux de la Cour.

Si la représentation nationale jouit d'informations fiables et impartiales en raison de l'indépendance de la Cour, celle-ci assistera d'autant mieux le Parlement que ses travaux lui seront utiles. Et sous cet angle, il importe pour la Cour qu'une large publicité soit accordée à ses enquêtes, analyses et conclusions. Les commissions des finances ou en charge des affaires sociales n'ont d'ailleurs jamais rechigné à publier les travaux de la Cour et à leur assurer une publicité minimale.

Ce lien de confiance établi a considérablement renforcé la qualité de l'assistance de la Cour au Parlement. D'où cette question: faut-il aller plus loin et élargir encore le périmètre de l'assistance, par exemple aux prévisions économiques et financières inscrites en loi de finances initiale ? Cette question est centrale dans la mesure où la portée d'un contrôle budgétaire a posteriori portant sur les résultats ne prend toute sa dimension que si les prévisions initiales ont été pertinentes. Or, de façon récurrente, les évaluations financières en loi de finances initiale sont toujours surestimées en termes de croissance et donc de recettes, et trop fréquemment sous-estimées en termes de dépenses, ce qui fausse l'évaluation des politiques publiques, leur pertinence, leur performance. Elles nuisent de la sorte à l'efficacité du contrôle de la représentation nationale. La proposition qui consiste à soulager le travail de l'Insee ${ }^{4}$ en impliquant la Cour des comptes objectiverait, par l'indépendance dont elle jouit, les hypothèses économiques et financières en loi de finances initiale, et par conséquent la pertinence du contrôle parlementaire a posteriori. Bien qu'alléchante, la suggestion n'est pas non plus dénuée de risque. Elle constituerait une charge supplémentaire pour la Cour, motif avancé par les sénateurs en juillet 2011 pour rejeter une disposition introduite par les députés dans la loi de finances rectificative (art. 64) consistant à permettre à un député de saisir la Cour d'une demande sur les suites données à ses recommandations en prolongement de la présentation des suites données aux observations des juridictions financières dans le rapport public. Qu'adviendrait-il par ailleurs si la Cour, comme l'administration, établissait des prévisions inexactes ? Le discrédit en serait jeté sur elle, affectant ses autres missions qui donnent totalement satisfaction à son «client» parlementaire.

La discussion mérite d'être ouverte, mais elle doit surtout conduire à une réflexion approfondie sur les outils de prévision à disposition du

4. Institut national de la statistique et des études économiques. 
gouvernement et le traitement politique qu'entend leur donner l'exécutif. Mais c'est là ouvrir un autre chapitre... sensible et pourtant décisif et primordial.

Malgré l'ancienneté des relations entre le Parlement et la Cour des comptes, ce n'est qu'au cours des deux dernières décennies que celles-ci se sont densifiées, traduisant de fait une alliance objective entre les deux institutions qui se renforcent ainsi mutuellement. Le Parlement profite de la qualité des travaux de la Cour pour intensifier avec pertinence son contrôle de l'action gouvernementale. La Cour, indépendante et réactive, gagne en respectabilité et se place comme une auxiliaire privilégiée $d u$ Parlement dans la reconquête de son autorité face à l'exécutif. 